\title{
Implementation of optimal phase-covariant cloning machines
}

\author{
Fabio Sciarrino ${ }^{1,2}$, and Francesco De Martini ${ }^{2}$ \\ ${ }^{1}$ Centro di Studi e Ricerche "Enrico Fermi", Via Panisperna 89/A, \\ Compendio del Viminale, Roma 00184, Italy \\ ${ }^{2}$ Dipartimento di Fisica and Consorzio Nazionale Interuniversitario per \\ le Scienze Fisiche della Materia, \\ Universitá "La Sapienza", Roma \\ 00185, Italy
}

\begin{abstract}
The optimal phase covariant cloning machine (PQCM) broadcasts the information associated to an input qubit into a multi-qubit systems, exploiting a partial a-priori knowledge of the input state. This additional a priori information leads to a higher fidelity than for the universal cloning. The present article first analyzes different experimental schemes to implement the $1 \longrightarrow 3 P Q C M$. The method is then generalized to any $1 \longrightarrow M$ machine for odd value of $M$ by a theoretical approach based on the general angular momentum formalism. Finally different experimental schemes based either on linear or non-linear methods and valid for single photon polarization encoded qubits are discussed.
\end{abstract}

The problem of manipulating and controlling the flux of quantum information between many quantum systems has in general been tackled and solved by the theory of quantum cloning and broadcasting [1, 2, 3]. From a practical point of view, this feature renders the theory of cloning a fundamental tool for the analysis of the security of quantum cryptographic protocols, for the distribution of quantum information to many partners and for the transmission of information contained in a system into correlations between many systems. In spite of the fact that, for fundamental reasons, the quantum cloning and flipping operations over an unknown qubit $|\phi\rangle$ are unrealizable in their exact forms [4, 5], they can be optimally approximated by the corresponding universal quantum machines, i.e. the universal optimal quantum cloning machine (UQCM) and the universal-NOT (U-NOT) gate [6]. The optimal quantum cloning machine has been experimentally realized following different approaches: by exploiting the process of stimulated emission [7, [8, 9], by means of a quantum network [10] and by adopting projective operators into the symmetric subspaces of many qubits [11, 12, 13]. The $N \rightarrow M$ UQCM transforms $N$ input qubits in the state $|\phi\rangle$ into $M$ output qubits, each one in the same mixed state $\rho_{\text {out }}$. The quality of the copies is quantified by the fidelity parameter $\mathcal{F}_{\text {univ }}^{N \rightarrow M}=\left\langle\phi\left|\rho_{\text {out }}\right| \phi\right\rangle=\frac{N+1+\beta}{N+2}$ with $\beta=\frac{N}{M} \leq 1$.

Not only the "universal" cloning of any unknown qubit is forbidden, but also the cloning of subsets containing non orthogonal states. This no-go theorem ensures the security of cryptographic protocols as $B B 84$ [14]. Recently state dependent, non universal, optimal cloning machines have been investigated where the cloner is optimal with respect to a given ensemble 15. This partial $a-$ priori knowledge of the state allows to reach a higher fidelity than for the universal cloning. The simplest and most relevant case is represented by the cloning covariant under the Abelian group $U(1)$ of phase rotations, the so called "phase-covariant" cloning. There the information is encoded in the phase $\phi_{i}$ of the input qubit belonging to any equatorial plane $i$ of the corresponding Bloch sphere.
In this context the general state may be expressed as: $\left|\phi_{i}\right\rangle=\left(\left|\psi_{i}\right\rangle+\exp \left(i \phi_{i}\right)\left|\psi_{i}^{\perp}\right\rangle\right)$ and $\left\{\left|\psi_{i}\right\rangle,\left|\psi_{i}^{\perp}\right\rangle\right\}$ is a convenient normalized basis, $\left\langle\psi_{i} \mid \psi_{i}^{\perp}\right\rangle=0$ [15]. Precisely, in the general case the $N \rightarrow M$ phase covariant cloning map $C_{N M}$ satisfies the following covariance relation $C_{N M}\left(T_{\phi i}^{\otimes N} \rho_{N} T_{\phi i}^{\dagger \otimes N}\right)=T_{\phi i}^{\otimes M} C_{N M}\left(\rho_{N}\right) T_{\phi i}^{\dagger \otimes M}$ where $T_{\phi i}=\exp \left[-\frac{i}{2} \phi_{i} \sigma_{i}\right]$. There the $\sigma_{i}$ Pauli operator identifies the set of input states which are cloned, e.g. $\sigma_{Y}$ corresponding to states belonging to the $x-z$ plane of the Bloch sphere. The values of the optimal fidelities $\mathcal{F}_{c o v}^{N \rightarrow M}$ for this machine have been found [16]. Restricting the analysis to a single input qubit to be cloned $N=1$ into $M>1$ copies, as we do in the present paper, the "cloning fidelity" is found: $\mathcal{F}_{\text {cov }}^{1 \rightarrow M}=\frac{1}{2}\left(1+\frac{M+1}{2 M}\right)$ for $M$ assuming odd values, or $\mathcal{F}_{\text {cov }}^{1 \rightarrow M}=\frac{1}{2}\left(1+\frac{\sqrt{M(M+2)}}{2 M}\right)$ for $M$ even. In particular we have $\mathcal{F}_{\text {cov }}^{1 \rightarrow 2}=0.854$ and $\mathcal{F}_{\text {cov }}^{1 \rightarrow 3}=0.833$ to be compared with the corresponding figures valid for universal cloning: $\mathcal{F}_{\text {univ }}^{1 \rightarrow 2}=0.833$ and $\mathcal{F}_{\text {univ }}^{1 \rightarrow 3}=0.778$.

In the above perspective it is worthwhile to enlighten the deep connection between the cloning processes and the theory of quantum measurement [17]. Indeed the concept of universal quantum cloning is related to the problem of optimal quantum state estimation since, for $M \rightarrow \infty$, and $\beta \longrightarrow 0$ the cloning fidelity converges toward the fidelity of state estimation of an arbitrary unknown qubit: $\mathcal{F}_{\text {univ }}^{N \rightarrow M} \rightarrow \mathcal{F}_{\text {estim }}^{N}=\frac{N+1}{N+2}[18$. In a similar way, the phase-covariant cloning is connected with the estimation of an equatorial qubit, that is, with the problem to find the optimal strategy to estimate the value of the phase $\phi[19,20]$. The optimal strategy has been found in [20]: it consists of a POVM measurement corresponding to a von Neuman measurement onto the $N$ input qubits characterized by a set of $N+1$ orthogonal projectors and achieves a fidelity $\mathcal{F}_{\text {phase }}^{N}$. In general for $M \rightarrow \infty, \mathcal{F}_{\text {cov }}^{N \rightarrow M} \rightarrow \mathcal{F}_{\text {phase }}^{N}$. In particular we have $\mathcal{F}_{\text {cov }}^{1 \rightarrow M}=\mathcal{F}_{\text {phase }}^{1}+\frac{1}{4 M}$ with $\mathcal{F}_{\text {phase }}^{1}=3 / 4$.

Recently the experimental realization of the $1 \rightarrow 3$ PQCM has been reported by adopting the methods of 
quantum optics 21]. The present article introduces in Section I different alternative approaches to implement the $1 \rightarrow 3$ device within any quantum information technique. In Section II such methods are generalized to any $1 \rightarrow M$ PQCM machine for odd value of $M$. There the corresponding theoretical analysis based by on the well established $\left|J, J_{z}\right\rangle$ angular momentum formalism of a general $J$-spin system will be given. Finally, in Section III different experimental schemes that can be adopted for single photon polarization encoded qubit based either on linear and non-linear methods will be presented.

\section{REALIZATION OF THE $1 \rightarrow 3$ PHASE-COVARIANT CLONING MACHINE}

In the present Section we describe two different techniques to implement the $1 \rightarrow 3$ PQCM. (a) The first method combines the implementation of a $1 \rightarrow 2 \mathrm{UQCM}$, together with a spin flipper $\sigma_{i}$ and the projection of the output qubits over the symmetric subspace: Fig. 1-(a). (b) The second one exploits the symmetrization of the input qubit to clone with an ancillary entangled pair: Fig. 1-(b).

We describe the approach (a), first introduced in 21]. The input qubit is expressed as: $|\phi\rangle_{S}=2^{-\frac{1}{2}}\left(|R\rangle_{S}+\right.$ $\left.\exp \left(i \phi_{Y}\right)|L\rangle_{S}\right)=\alpha|0\rangle_{S}+\beta|1\rangle_{S}$, with $\langle R \mid L\rangle=0,|\alpha|^{2}+$ $|\beta|^{2}=1$ and $\alpha, \beta$ real parameters. Here we consider, in particular, the $\phi_{Y}$-covariant cloning and $\sigma_{i}=\sigma_{Y}$ realizes the NOT gate for the qubits belonging to the $x-z$ plane. The output state of the $1 \rightarrow 2$ UQCM device reads:

$$
\begin{aligned}
& |\Sigma\rangle_{S A B}=\sqrt{\frac{2}{3}}|\phi\rangle_{S}|\phi\rangle_{A}\left|\phi^{\perp}\right\rangle_{B} \\
& -\frac{1}{\sqrt{6}}\left(|\phi\rangle_{S}\left|\phi^{\perp}\right\rangle_{A}+\left|\phi^{\perp}\right\rangle_{S}|\phi\rangle_{A}\right)|\phi\rangle_{B}
\end{aligned}
$$

where the qubits $S$ and $A$ are the optimal cloned qubits while the qubit $B$ is the optimally flipped one. According to the scheme represented by Fig. 1-(a), the idea is now to exactly flip the qubit $B$ for a given subset of the Bloch sphere. This local flipping transformation of $|\phi\rangle_{B}$ leads to: $|\Upsilon\rangle_{S A B}=\left(I_{S} I_{A} \otimes \sigma_{Y}\right)|\Sigma\rangle_{S A B}=\sqrt{\frac{2}{3}}|\phi\rangle_{S}|\phi\rangle_{A}|\phi\rangle_{B}+$ $-\frac{1}{\sqrt{6}}\left(|\phi\rangle_{S}\left|\phi^{\perp}\right\rangle_{A}+\left|\phi^{\perp}\right\rangle_{S}|\phi\rangle_{A}\right)\left|\phi^{\perp}\right\rangle_{B}$. By this nonuniversal cloning process three asymmetric copies have been obtained: two clones (qubits $S$ and $A$ ) with fidelity $5 / 6$, and a third one (qubit $B$ ) with fidelity $2 / 3$. We may now project $S, A$ and $B$ over the symmetric subspace and obtain three symmetric clones with a higher average fidelity. The symmetrization operator $\Pi_{S A B}^{3}$ reads as $\Pi_{S A B}^{3}=$ $\left|\Pi_{1}\right\rangle\left\langle\Pi_{1}|+| \Pi_{2}\right\rangle\left\langle\Pi_{2}|+| \Pi_{3}\right\rangle\left\langle\Pi_{3}|+| \Pi_{4}\right\rangle\left\langle\Pi_{4}\right|$ where $\left|\Pi_{1}\right\rangle=$ $|\phi\rangle_{S}|\phi\rangle_{A}|\phi\rangle_{B}, \quad\left|\Pi_{2}\right\rangle=\left|\phi^{\perp}\right\rangle_{S}\left|\phi^{\perp}\right\rangle_{A}\left|\phi^{\perp}\right\rangle_{B},\left|\Pi_{3}\right\rangle=$ $\frac{1}{\sqrt{3}}\left(|\phi\rangle\left|\phi^{\perp}\right\rangle\left|\phi^{\perp}\right\rangle+\left|\phi^{\perp}\right\rangle|\phi\rangle\left|\phi^{\perp}\right\rangle+\left|\phi^{\perp}\right\rangle\left|\phi^{\perp}\right\rangle|\phi\rangle\right)$ and $\left|\Pi_{4}\right\rangle=\frac{1}{\sqrt{3}}\left(|\phi\rangle|\phi\rangle\left|\phi^{\perp}\right\rangle+\left|\phi^{\perp}\right\rangle|\phi\rangle|\phi\rangle+|\phi\rangle\left|\phi^{\perp}\right\rangle|\phi\rangle\right)$. The symmetric subspace has dimension 4 since three

\section{(a)}
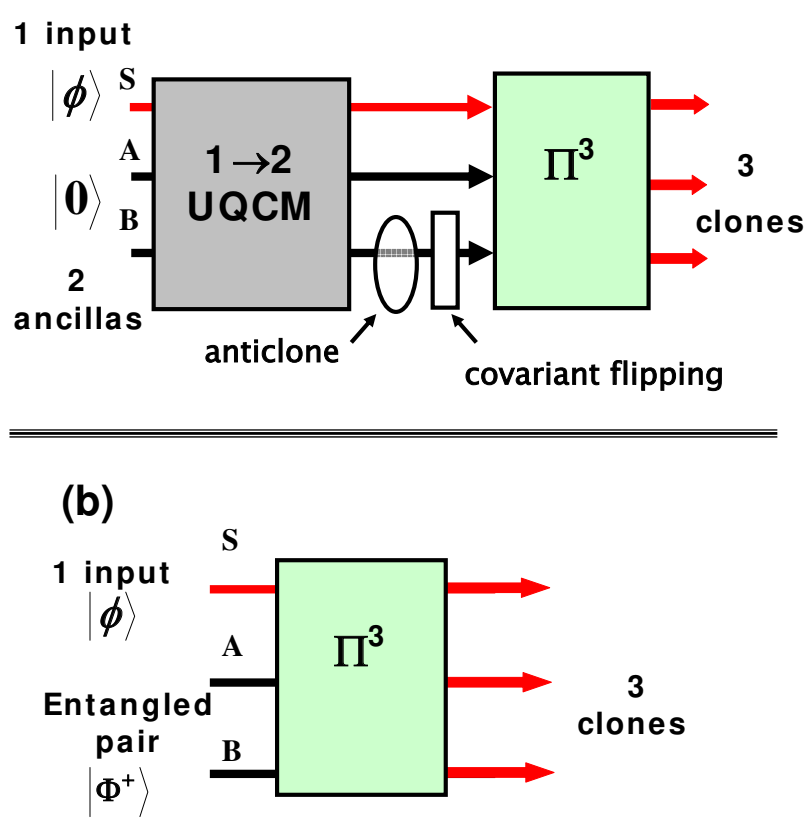

FIG. 1: Scheme for the realization of the $1 \rightarrow 3$ PQCM. (a) UQCM, phase-covariant cloning and projection of the output state over the symmetric subspace $\Pi^{3}$. (b) Symmetrization process acting on the input qubit and one entangled pair of qubits.

qubits are involved. The probability of success of the projection is equal to $\frac{8}{9}$. The normalized output state $|\xi\rangle_{S A B}=\Pi_{S A B}^{3}|\Upsilon\rangle_{S A B}$ is

$$
|\xi\rangle_{S A B}=\frac{1}{2} \sqrt{3}\left[|\phi\rangle_{S}|\phi\rangle_{A}|\phi\rangle_{B}-3^{-1}\left(|\phi\rangle_{S}\left|\phi^{\perp}\right\rangle_{A}\left|\phi^{\perp}\right\rangle_{B}+\left|\phi^{\perp}\right\rangle_{S}|\phi\rangle_{A}\left|\phi^{\perp}\right\rangle_{B}+\left|\phi^{\perp}\right\rangle_{S}\left|\phi^{\perp}\right\rangle_{A}|\phi\rangle_{B}\right)\right]
$$


$\frac{1}{6}\left|\phi^{\perp}\right\rangle\left\langle\phi^{\perp}\right|$. This leads to the fidelity $\mathcal{F}_{\text {cov }}^{1 \rightarrow 3}=5 / 6$ equal to the optimal one obtained in the general case [15, 16]. By applying a different unitary operator $\sigma_{i}$ to the qubit $B$ we can implement the phase-covariant cloning for the corresponding different equatorial planes of the Bloch sphere, orthogonal to the $i$-axis.

Let us now consider the second approach (b), which represents an innovative simplification of the previous scheme. The PQCM device can be realized by applying the symmetrization projection $\Pi_{S A B}^{3}$ to the input qubit and to an ancillary entangled pair $\left|\Phi^{+}\right\rangle_{A B}=$ $\frac{1}{\sqrt{2}}\left(|0\rangle_{A}|0\rangle_{B}+|1\rangle_{A}|1\rangle_{B}\right)$. The output state reads:

$$
\Pi_{S A B}^{3}\left(|\phi\rangle_{S} \otimes\left|\Phi^{+}\right\rangle_{A B}\right)=|\xi\rangle_{S A B}
$$

Again the qubits $S, A$ and $B$ are found to be the optimal phase-covariant clones of the input one. By modifying the ancillary entangled state, the set of states cloned is changed. The state $\left|\Psi^{+}\right\rangle_{A B}$ leads to the PQCM machine for the $y-z$ plane, while $\left|\Phi^{-}\right\rangle_{A B}$ for the $x-y$ plane. Such result is at variance with the one found for the universal cloning process [12]. Indeed the $1 \rightarrow 3$ UQCM transformation can be achieved by applying the projector $\Pi_{S A B}^{3}$ to the qubit $|\phi\rangle_{S}$ and to two ancillas qubit, each one in a fully mixed state $\frac{I}{2}$.

\section{GENERAL APPROACH: $1 \rightarrow \mathrm{M}$ DEVICE}

In the present Section the two previous approaches are generalized to the realization of the $1 \rightarrow M=2 P-1$ PQCM: the first one (a) exploits the universal cloning machine, covariant flipping and final symmetrization while the second one (b) is based on appropriate symmetrization of the input qubit with entangled pairs of qubit.

Let us consider the scheme of Fig.2-(a). The UQCM broadcast the information on the input qubit over $2 P-1$ qubit. The overall output state after the UQCM map reads

$\left|\Omega^{\prime}\right\rangle=\sum_{k=0}^{P-1} b_{k}\left|\left\{(P-k) \phi ; k \phi^{\perp}\right\}\right\rangle_{C} \otimes\left|\left\{k \phi ;(P-1-k) \phi^{\perp}\right\}\right\rangle_{A C}$

where $b_{k}=(-1)^{k} \sqrt{\frac{2}{P+1}} \sqrt{\frac{(P-1) !(P-k) !}{P !(P-1-k) !}}$ and the notation $\left|\left\{p \phi ; q \phi^{\perp}\right\}\right\rangle$ stands for a total symmetric combination of $p$ qubits in the state $|\phi\rangle$ and of $q$ qubits in the state $\left|\phi^{\perp}\right\rangle[6]$. The labels $C$ and $A C$ identify, respectively, the cloning and anticloning subsystems. Hereafter, we assume the input qubit to be in the state $|\phi\rangle=|0\rangle$ without lack of generality. The $P$ qubits of the set $C$ exhibit a fidelity of the cloning process equal to $\mathcal{F}_{1 \rightarrow P}=\frac{2+\beta}{3}$ with $\beta=1 / P$, while the $P-1$ qubits of the set $A C$ exhibit a fidelity of the flipping process equal to $\mathcal{F}_{1 \rightarrow(P-1)}^{*}=\frac{2}{3}$. We associate to each qubit state a spin $\frac{1}{2}$ system. The previous expression can hence be expressed by exploiting the formalism of the angular momentum $\left|J, J_{z}\right\rangle$ of a (a)

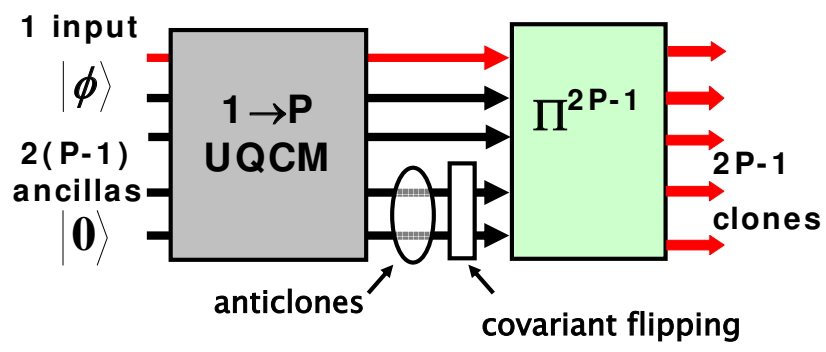

(b)

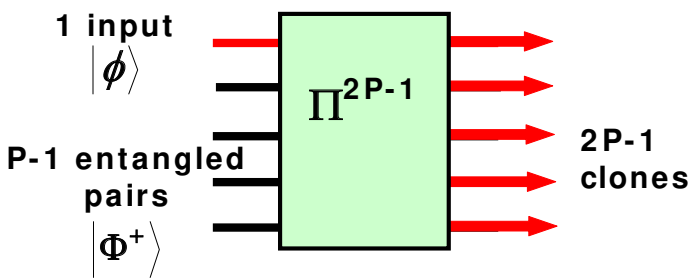

FIG. 2: General scheme for the realization of the $1 \rightarrow(2 P-$ 1) PQCM. (a) $1 \rightarrow P$ UQCM, phase-covariant cloning and projection of the output state over the symmetric subspace $\Pi^{2 P-1}$. (b) Symmetrization process acting on the input qubit and $(P-1)$ entangled pairs of qubits.

general $J$-spin system. The overall state in the basis $\left|j ; m_{j}\right\rangle_{C} \otimes\left|j ; m_{j}\right\rangle_{A C}$ reads

$\left|\Omega^{\prime}\right\rangle=\sum_{k=0}^{P-1} b_{k}\left|\frac{P}{2} ; \frac{P}{2}-k\right\rangle_{C} \otimes\left|\frac{P-1}{2} ; \frac{-(P-1)}{2}+k\right\rangle_{A C}$

In the above representation, the overall output state of the cloner is written as the composition of two angular momenta: $\mathbf{J}_{C}, \mathbf{J}_{A C}$ defined respectively over the "cloning" and "anticloning" output channels. We note that the qubits $A C$ assume the maximum allowed value of $J=\frac{P-1}{2}$, thus they lie in the symmetric subspace in analogy with the clone ones.

As following step, a covariant flipping process is ap- 
plied to the subspace $A C$ transforming $\left|\Omega^{\prime}\right\rangle$ into

$$
\begin{aligned}
& \left|\Omega^{\prime \prime}\right\rangle=I_{C} \otimes\left(\sigma_{Y}^{\otimes(P-1)}\right)_{A C}\left|\Omega^{\prime}\right\rangle \\
& =\sum_{k=0}^{P-1} b_{k}\left|\frac{P}{2} ; \frac{P}{2}-k\right\rangle_{C} \otimes\left|\frac{P-1}{2} ; \frac{(P-1)}{2}-k\right\rangle_{A C}
\end{aligned}
$$

Such expression holds for any qubit belonging to the equatorial plane under consideration. Let us now express $\left|\Omega^{\prime \prime}\right\rangle$ adopting the overall angular momentum $\mathbf{J}_{T}=\mathbf{J}_{C}+$ $\mathbf{J}_{A C}$ in the basis $\left|j_{C} ; j_{A C} ; j_{T} ; m_{T}\right\rangle$

$$
\left|\Omega^{\prime \prime}\right\rangle=\sum_{j_{T}=1 / 2}^{2 P-1} \sum_{m_{T}=-j_{T}}^{j_{T}} c\left(j_{T}, m_{T}\right)\left|\frac{P}{2} ; \frac{P-1}{2} ; j_{T} ; m_{T}\right\rangle
$$

where $c\left(j_{T}, m_{T}\right)$ can be derived exploiting the Clebsch - Gordan coefficient $\left\langle j_{1} ; j_{2} ; m_{1} ; m_{2} \mid j_{1} ; j_{2} ; j_{T} ; m_{T}\right\rangle$ with $j_{1}=\frac{P}{2}, j_{2}=\frac{P-1}{2}, m_{1 k}=\frac{P}{2}-k, m_{2 k}=\frac{(P-1)}{2}-k$ 22].

To complete the protocol, the overall output state is symmetrized by applying the projector $\Pi^{M}$ with $M=2 P-1$ defined as: $\Pi^{M}=\sum_{j=0}^{M}\left|\frac{P}{2} ; \frac{P-1}{2} ; \frac{M}{2} ; \frac{M}{2}-j\right\rangle\left\langle\frac{P}{2} ; \frac{P-1}{2} ; \frac{M}{2} ; \frac{M}{2}-j\right|$. The non-vanishing contributions to the projected state comes from terms with $j_{T}=\frac{2 P-1}{2}$. After the action of $\Pi^{M}$ we obtain the following normalized output state

$$
\left|\Omega^{\prime \prime \prime}\right\rangle=\Pi^{M}\left|\Omega^{\prime \prime}\right\rangle=\sum_{k=0}^{P-1} d_{k}\left|\frac{P}{2} ; \frac{P-1}{2} ; \frac{2 P-1}{2} ; \frac{2 P-1}{2}-2 k\right\rangle
$$

with

$$
\begin{aligned}
d_{k} & =b_{k}\left\langle\frac{P}{2} ; \frac{P-1}{2} ; \frac{P}{2}-k ; \frac{(P-1)}{2}-k \mid \frac{P}{2} ; \frac{P-1}{2} ; \frac{2 P-1}{2} ; \frac{2 P-1}{2}-2 k\right\rangle= \\
& =(-1)^{k} \sqrt{\frac{2}{P+1}}\left(\begin{array}{c}
P-1 \\
k
\end{array}\right)\left(\begin{array}{c}
2 P-1 \\
2 k
\end{array}\right)^{-1 / 2}
\end{aligned}
$$

The normalization factor reads

$$
\left.\left|\Pi^{M}\right| \Omega^{\prime \prime}\right\rangle\left.\right|^{2}=\frac{2}{P+1} \sum_{k=0}^{P-1} \frac{\left(\begin{array}{c}
P-1 \\
k
\end{array}\right)^{2}}{\left(\begin{array}{c}
2 P-1 \\
2 k
\end{array}\right)}
$$

The fidelities of the phase-covariant cloning process can be inferred re-arranging the output state (8) as follows

$$
\left|\Omega^{M}\right\rangle=\sum_{k=0}^{2 P-1} d_{k}\left|\left\{(2 P-1-2 k) \phi ; 2 k \phi^{\perp}\right\}\right\rangle
$$

All the $2 P-1$ qubits belonging to such state have an identical reduced density matrix equal to

$$
\rho_{\text {cov }}=\gamma(P)|\phi\rangle\left\langle\phi|+(1-\gamma(P))| \phi^{\perp}\right\rangle\left\langle\phi^{\perp}\right|
$$

with

$$
\gamma(P)=\frac{\sum_{k=0}^{P-1} \frac{(2 P-1-2 k)}{(2 P-1)} \frac{\left(\begin{array}{c}
P-1 \\
k
\end{array}\right)^{2}}{\left(\begin{array}{c}
2 P-1 \\
2 k
\end{array}\right)}}{\sum_{k=0}^{P-1} \frac{\left(\begin{array}{c}
P-1 \\
k
\end{array}\right)^{2}}{\left(\begin{array}{c}
2 P-1 \\
2 k
\end{array}\right)}}=\frac{1}{2}\left(1+\frac{M+1}{2 M}\right)
$$

The previous expression has been demonstrated numerically, for value of $M$ up to 2000 .
The fidelity of the cloning process is thus

$$
\mathcal{F}_{1 \rightarrow M}=\left\langle\phi\left|\rho_{\text {cov }}\right| \phi\right\rangle=\frac{1}{2}\left(1+\frac{M+1}{2 M}\right)
$$

and is found equal to the optimal one.

As alternative approach, the $1 \rightarrow M$ PQCM device can be obtained by applying the symmetrization projector $\Pi^{M}$ over the input qubit and $(P-1)$ ancilla entangled pairs $\left|\Phi^{+}\right\rangle_{A B}$ : Fig.2-(b). Such a result can easily be obtained by manipulating the scheme of Fig.2-(a) as follows. The UQCM of Fig. 2-(a) can be realized starting from the input qubit $|\phi\rangle$ and $(P-1)$ entangled pairs $\left|\Psi^{-}\right\rangle_{A B}$ as shown in Ref. 12]. The cloning map is achieved by symmetrization of the input qubit and $(P-1)$ ancilla qubits $A$, each one belonging to an entangled pair $\left|\Psi^{-}\right\rangle_{A B}$

$$
\Pi_{S A}^{P} \otimes I_{B}^{P-1}\left(|\phi\rangle_{S}\left|\Psi^{-}\right\rangle_{A B}^{\otimes(P-1)}\right)
$$

The output state is equal to the one $\left|\Omega^{\prime}\right\rangle$ of Eq[5] up to a normalization factor. To implement the PQCM device, the covariant flipping $\sigma_{Y}$ is then applied to the $(P-1)$ qubits belonging to the subset $B$. The same result can 
be obtained starting from the input state $\left|\Phi^{+}\right\rangle_{A B}^{\otimes(P-1)}$, indeed

$$
\begin{aligned}
& \left(I_{S A}^{P} \otimes \sigma_{Y-B}^{\otimes(P-1)}\right)\left(\Pi_{S A}^{P} \otimes I_{B}^{P-1}\left(|\phi\rangle_{S}\left|\Psi^{-}\right\rangle_{A B}^{\otimes(P-1)}\right)\right) \\
& =\left(\Pi_{S A}^{P} \otimes I_{B}^{P-1}\left(|\phi\rangle_{S}\left|\Phi^{+}\right\rangle_{A B}^{\otimes(P-1)}\right)\right)
\end{aligned}
$$

As final step the overall state is projected into the symmetric subspace through the projector $\Pi_{S A B}^{2 P-1}$ :

$$
\begin{aligned}
\left|\Omega^{\prime \prime \prime}\right\rangle & =\Pi_{S A B}^{2 P-1}\left(\left(\Pi_{S A}^{P} \otimes I_{B}^{P-1}\left(|\phi\rangle\left|\Phi^{+}\right\rangle_{A B}^{\otimes(P-1)}\right)\right)\right)(16) \\
& =\Pi_{S A B}^{2 P-1}\left(|\phi\rangle_{S}\left|\Phi^{+}\right\rangle_{A B}^{\otimes(P-1)}\right)
\end{aligned}
$$

In the previous expression we have exploited the concatenation property of the symmetrization projector $\Pi_{S A B}^{2 P-1}\left(\Pi_{S A}^{P} \otimes I_{B}^{P-1}\right)=\Pi_{S A B}^{2 P-1}$ which has been demonstrated experimentally in Ref. [23]. This concludes our simple proof of the scheme of Fig.2-(b).

\section{REALIZATION BY QUANTUM OPTICS}

In quantum optics the qubit can be implemented by exploiting the isomorphism between the qubit state $|\phi\rangle=$ $\alpha|0\rangle+\beta|1\rangle$ and the polarization state $\alpha|H\rangle+\beta|B\rangle$ of a single photon. In this context it has been proposed to realize the unitary transformation, $U_{N \rightarrow M}$, leading to the deterministic UQCM, by means of the "quantum injected" optical parametric amplification (QIOPA) in the entangled configuration. The experimental demonstrations of both optimal cloning and flipping processes by exploiting this technique have been reported in 8, 12, 13]. At the same time, a different scenario has been disclosed by the discovery that it is possible to implement contextually the $1 \rightarrow 2$ universal quantum cloning machine (UQCM) and the $1 \rightarrow 1$ universal NOT gate by modifying the quantum state teleportation protocol [1, 12]. The last procedure is based on a symmetric projective operation realized by combining single-photon interferometry and post-selection techniques, and it can be extended to the generic $N \rightarrow M$ cloning device.

The symmetrization of two polarization encoded qubit can be achieved by letting two independent-qubits to impinge onto the input arms of a beam splitter (BS) in an Hong-Ou-Mandel interferometer [24], and then by probabilistically post-selecting the events in which the two photons emerge in the same spatial output mode. The basic principle at the heart of these realizations is the following: the two photons are initially superimposed at the BS interface in order to make them indistinguishable; then, a spatial symmetric wavefunction of the two photons is post-selected by the measurement apparatus. Such scheme can be extended in a controlled way to an higher number of photons, as shown in Ref. 23]. There a linear optics multi qubit symmetrization apparatus has been realized by a chain of interconnected Hong-Ou-Mandel interferometer.

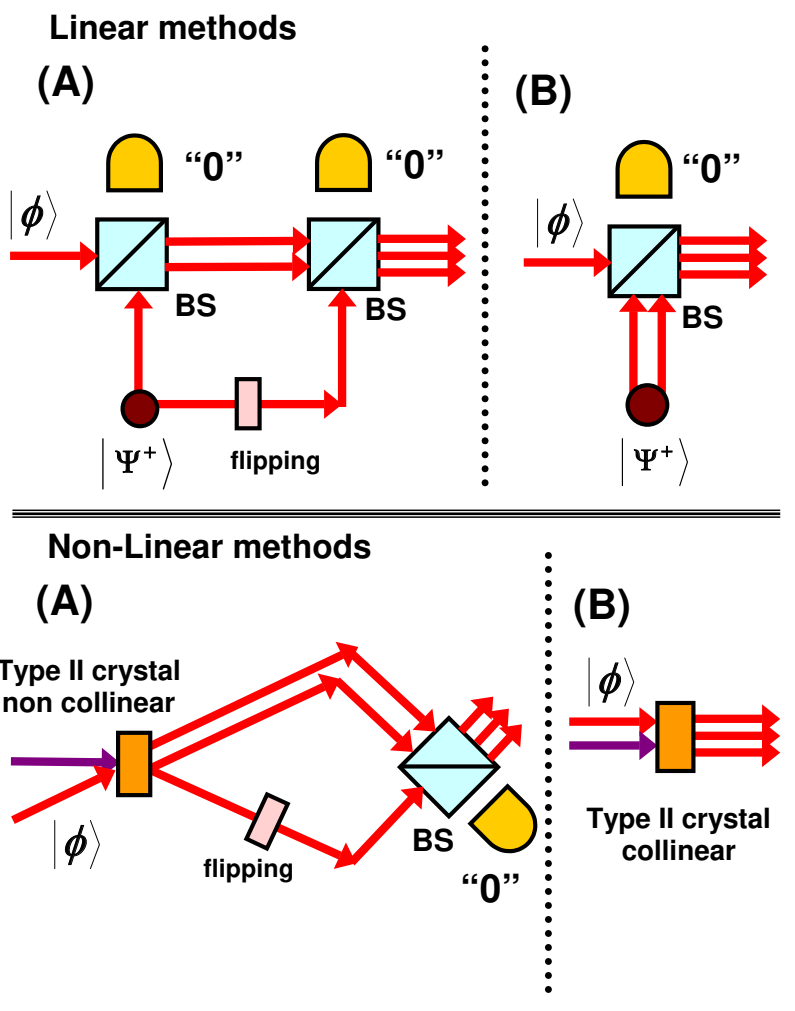

FIG. 3: Linear methods: (a) schematic diagram of the linear optics multi qubit symmetrization apparatus realized by a chain of interconnected Hong-Ou-Mandel interferometer; (b) symmetrization of the input photon and the ancilla polarization entangled pairs. Non-linear methods: (a) UQCM by optical parametric amplification, flipping by a couple of waveplates and projection over the symmetric subspace; (b) collinear optical parametric amplification within a type II BBO crystal.

Here we introduce a variety of schemes which can be realized through the methods of quantum optics outlined above. By restricting our attention to the $1 \rightarrow 3$ PQCM, the diagram below can be easily extended to general case $1 \rightarrow M$ for odd values of $M$ following the guidelines of the previous Section. Let us consider first linear optics approach. Fig.3 shows the experimental scheme implementing, respectively, the scheme of Fig.1-(a), (A), and Fig.1-(b), (B). The flipping operation $\sigma_{Y}$ is realized by means of two $\lambda / 2$ waveplates acting on the polarization state, while the symmetrization is implemented by overlapping the incoming photons on a beam splitter and post-selecting the events in which they emerge over the same mode, as said. Such scheme is similar to the one proposed by Zou et al. 25] to implement the $1 \rightarrow 3$ PQCM for photonic qubit.

Finally the same results can be obtained adopting nonlinear methods. Let us consider the $1 \rightarrow 3$ PQCM, in 
particular the optimal quantum cloning for $x-z$ equatorial qubits by taking linear polarization states as input. The $U Q C M$ has been realized by adopting a quantuminjected optical parametric amplifier (QIOPA), while the $\sigma_{Y}$ operation and the $\Pi^{3}$ projection have been implemented with linear optics and post-selection techniques Fig.(a). The flipping operation on the output mode $\mathbf{k}_{A C}$ was realized by means of two $\lambda / 2$ waveplates, while the physical implementation of the projector $\Pi^{3}$ on the three photons-states was carried out by linearly superimposing the modes $\mathbf{k}_{C}$ and $\mathbf{k}_{A C}$ on the 50:50 beamsplitter $B S$ and then by selecting the case in which the three photons emerged from $B S$ on the same output mode $\mathbf{k}_{P C}$ (or, alternatively on $\mathbf{k}_{P C}^{\prime}$ ).

Interestingly, the same overall state evolution can also be obtained, with no need of the final $B S$ symmetrization, at the output of a QI-OPA with a type II crystal working in a collinear configuration, (b) 26]. In this case the interaction Hamiltonian $\widehat{H}_{\text {coll }}=i \chi \hbar\left(\widehat{a}_{H}^{\dagger} \widehat{a}_{V}^{\dagger}\right)+$ h.c. acts on a single spatial mode $k$. A fundamental physical property of $\widehat{H}_{\text {coll }}$ consists of its rotational invariance under $U(1)$ transformations, that is, under any arbitrary rotation around the z-axis. Indeed $\widehat{H}_{\text {coll }}$ can be re-expressed as $\frac{1}{2} i \chi \hbar e^{-i \phi}\left(\widehat{a}_{\phi}^{\dagger 2}-e^{i 2 \phi} \widehat{a}_{\phi \perp}^{\dagger 2}\right)+h . c$. for $\phi \in(0,2 \pi)$ where $\widehat{a}_{\phi}^{\dagger}=2^{-1 / 2}\left(\widehat{a}_{H}^{\dagger}+e^{i \phi} \widehat{a}_{V}^{\dagger}\right)$ and $\widehat{a}_{\phi \perp}^{\dagger}=$ $2^{-1 / 2}\left(-e^{-i \phi} \widehat{a}_{H}^{\dagger}+\widehat{a}_{V}^{\dagger}\right)$. Let us consider an injected single photon with polarization state $|\phi\rangle_{i n}=2^{-1 / 2}(|H\rangle+$ $\left.e^{i \phi}|V\rangle\right)=|1,0\rangle_{k}$ where $|m, n\rangle_{k}$ represents a product state with $m$ photons of the mode $k$ with polarization $\phi$, and $n$ photons with polarization $\phi^{\perp}$. The first contribution to the amplified state, $\sqrt{6}|3,0\rangle_{k}-\sqrt{2} e^{i 2 \phi}|1,2\rangle_{k}$ is identical to the output state obtained with the device introduced above up to a phase factor which does not affect the fidelity value.

\section{CONCLUSIONS}

We have introduced different schemes to implement the optimal $1 \rightarrow M>1$ phase covariant cloning machine, by exploiting either the QIOPA method or the projection over the symmetric subspace. The introduced approaches are probabilistic, however such feature does not spoil the main physical result of the present procedure since the optimal fidelity value can not be improved by any probabilistic procedure implementation [27]. The present schemes do not hold for even values of $M$. Indeed it has been noticed that different features affect the $1 \rightarrow 2 P$ and $1 \rightarrow(2 P-1)$ PQCM maps [15]. Recently an optical scheme to realize the $1 \rightarrow 2$ PQCM has been proposed [28] and realized experimentally [29].

The experimental realization of the different protocols with the standard quantum optics techniques has been discussed. There we found an answer to the question recently raised by Scarani et al. [1] whether it is possible implement any cloning transformation different from the universal one using amplification through stimulated emission. We have just seen that this can it be done directly either by linear optics elements, either by a nonlinear, quantum injected optical parametric amplification process. The generalization of such schemes to an higher number of input qubits $N>1$ has been found to be non-optimal and hence deserves further investigation.

Finally we shall enlighten that the present cloning maps are economical, that is, do not require any extra physical resources than the clones qubits [30].

We acknowledge financial support from the Ministero della Istruzione, dellUniversità e della Ricerca (PRIN 2005).
[1] V. Scarani, S. Iblisdir, N. Gisin, and A. Acín, Rev. Mod. Phys. 77, 1225 (2005).

[2] N. Cerf, and J. Fiurasek, quant-ph/0512172

[3] F. De Martini, and, F. Sciarrino, Progress in Quantum Electronics 29, 165 (2005).

[4] W.K. Wootters, and W.H. Zurek, Nature (London) 299, 802 (1982).

[5] H. Bechmann-Pasquinucci and N. Gisin, Phys. Rev. A 59, 4238 (1999).

[6] V. Bužek, and M. Hillery, Phys. Rev. A 54, 1844 (1996); N. Gisin, and S. Massar, Phys. Rev. Lett. 79, 2153 (1997); R. Derka, V. Buzek and A. Ekert, Phys. Rev. Lett. 80, 1571 (1998).

[7] F. De Martini, V. Bužek, F. Sciarrino, and C. Sias, Nature (London) 419, 815 (2002); D. Pelliccia, et al., Phys. Rev. A 68, 042306 (2003); F. De Martini, D. Pelliccia, and F. Sciarrino, Phys. Rev. Lett. 92, 067901 (2004).

[8] A. Lamas-Linares, C. Simon, J.C. Howell, and D. Bouwmeester, Science 296, 712 (2002).

[9] S. Fasel, et al., Phys. Rev. Lett. 89, 107901 (2002).
[10] H.K. Cummins et al., Phys. Rev. Lett. 88, 187901 (2002).

[11] M. Ricci, F. Sciarrino, C. Sias, and F. De Martini, Phys. Rev. Lett. 92, 047901 (2004); F. Sciarrino, C. Sias, M. Ricci, and F. De Martini, Phys. Lett. A 323, 34 (2004).

[12] F. Sciarrino, C. Sias, M. Ricci, and F. De Martini, Phys. Rev. A 70, 052305 (2004).

[13] W.T.M. Irvine, A. Lamas Linares, M.J.A. de Dood, and D. Bouwmeester, Phys. Rev. Lett. 92, 047902 (2004).

[14] N. Gisin, G. Ribordy, W. Tittel., H. Zbinden, Rev. Mod. Phys. 74, 145 (2002).

[15] D. Bru $\beta$, M. Cinchetti, G.M. D'Ariano, and C. Macchiavello, Phys. Rev. A 62, 012302 (2000); G.M. D'Ariano, and P. Lo Presti, Phys. Rev. A 64, 042308 (2001); H. Fan, K. Matsumoto, X. Wang, and M. Wadati, Phys. Rev. A 65, 012304 (2001).

[16] D. Bru $\beta$ and C. Macchiavello, J. Phys. A 34, 6815 (2001); G.M. D'Ariano, and C. Macchiavello, Phys. Rev. A 67, 042306 (2003).

[17] D. Bruss, A. Ekert, and C. Macchiavello, Phys. Rev. Lett. 81, 2598 (1998). 
[18] S. Massar and S. Popescu, Phys. Rev. Lett. 74, 1259 (1995).

[19] A.S. Holevo, Probabilistic and Statistical Aspects of Quantum Theory (North-Holland, Amsterdam, 1982), p.163.

[20] R. Derka, V. Buzek, and A.E. Ekert, Phys. Rev. Lett. 80, 1571 (1998).

[21] F. Sciarrino, and F. De Martini, Phys. Rev. A 72, 062313 (2005).

[22] A.R. Edmonds, Angular momentum in quantum mechanics (2. ed.) (N.Y.: Princeton University press, 1960).

[23] L. Masullo, M. Ricci, and F. De Martini, Phys. Rev. A
72, 060304 (2005).

[24] C.K. Hong, Z.Y. Ou, and L. Mandel, Phys. Rev. Lett. 59, 2044 (1987).

[25] X. Zou and W. Mathis, Phys. Rev. A 72, 022306 (2005)

[26] F. De Martini, Phys. Lett. A 250, 15 (1998).

[27] J. Fiurasek, Phys. Rev. A 70, 032308 (2004).

[28] J. Fiurasek, Phys. Rev. A 67, 052314 (2003).

[29] A. Cernoch, et al., quant-ph/0607149

[30] F. Buscemi, G.M.D'Ariano, C. Macchiavello, Phys. Rev. A 71, 042327 (2005); T. Durt, J.Fiurasek, N.J. Cerf, Phys. Rev. A 72, 052322 (2005). 